\title{
Absence status associated with focal activity and polydipsia-induced hyponatremia
}

\author{
Hideki Azuma \\ Tatsuo Akechi \\ Toshi A Furukawa \\ Department of Psychiatry and \\ Cognitive-Behavioral Medicine, \\ Nagoya City University Graduate \\ School of Medical Sciences, Nagoya, \\ Japan
}

\begin{abstract}
We report a case of de novo absence status associated with focal discharge and polydipsia-induced hyponatremia. Nonconvulsive status epilepticus (NCSE) is classified as absence status or complex partial status. Absence status is characterized by bilateral synchronized spike and wave complex bursts and a variety of conscious disturbances. Possible precipitating factors for NCSE include benzodiazepine withdrawal, excessive use of psychotropic drugs, and electrolyte imbalances. Hyponatremia is a rare precipitating factor. In this case, the patient was 59 years old and had suffered from primary insomnia but had no history of epilepsy. NCSE improved by means of saline infusion. However after recovery from NCSE EEG revealed some spikes in the left frontal area. Absence seizures can also show generalized spike and slow waves, and cases of focal lesion-associated absence seizures have been reported. Although absence seizures and absence status are two distinct conditions, they should not be considered together. We assumed that hyponatremia induced by polydipsia precipitated epileptogenicity in the left frontal area, and then focal activity secondarily generalized and resulted in absence status.
\end{abstract}

Keywords: nonconvulsive status epilepticus, absence status, focal activity, hyponatremia

\section{Introduction}

Nonconvulsive status epilepticus (NCSE) can be classified as absence status or complex partial status. Absence status is characterized by bilateral synchronized spike and wave complex bursts and a variety of conscious disturbances (Niedermeyer and Ribeiro 2000). Possible precipitating factors for NCSE include benzodiazepine withdrawal, excessive use of the psychotropic drugs, and electrolyte imbalances. Hyponatremia is a rare precipitating factor (Lee 1985; Thomas et al 1992; Tomson et al 1992). The standard treatment for polydipsia-induced hyponatremia is intravenous saline solution. Primavera et al reported that a case of NCSE resulting from hyponatremia, was improved with saline solution treatment (Primavera et al 1995).

Absence seizures are also characterized by generalized spike and slow wave complexes, and cases with focal lesion-associated absences have been reported (Millan et al 2001). However, absence seizure and absence status are distinct conditions that should be considered separately. Interestingly, we observed a case of de novo absence status associated with a region of focal brain activity, suspected of being an epileptogenic zone and hyponatremia caused by polydipsia.

\section{Case report}

Since X-2 year, a 57-year-old man had been treated for primary insomnia at a psychiatric clinic, and psychotropics were administered. He had developed the habit of drinking large amounts of water, about 2-3 liters per day at that time. His psychiatric and physical history was of no particular significance. He had no family history of seizure disorder, and no history of hypertension, diabetes, perinatal difficulty, stroke, or trauma. 
In April of X year, the patient began vomiting and could not say his own name, address, or the date. The next day he and his family presented at our psychiatric clinic. At first examination he showed poor orientation, memory disturbances, a decrease in spontaneous speech, bradykinesia, confusion, and was suspected to have a subacute consciousness disturbance. He was admitted to our psychiatric ward for further examination on that day. On admission he had taken etizolam $2 \mathrm{mg}$, sulpiride $150 \mathrm{mg}$, promethazine $12.5 \mathrm{mg}$, chlorpromazine $12.5 \mathrm{mg}$, and phenobarbital $15 \mathrm{mg}$. Head computed tomography showed only lacunars at the left basal ganglia. Electrolyte analysis revealed hyponatremia (118 mEq/L)(138-146 ), urine osmolarity $369 \mathrm{mOsm} / \mathrm{kg}$ (50-1300), urine sodium $0.85 \mathrm{~g} /$ day (4-6), serum osmolarity $268 \mathrm{mOsm} / \mathrm{kg}$ (275-290), and antidiuretic hormone (ADH) was $8.4 \mathrm{pg} / \mathrm{mL}(0.3-3.5)$, and we suspected the syndrome of inappropriate secretion of antidiuretic hormone (SIADH). No potential cardiac, hepatic, renal causes or paraneoplastic syndrome were detected. Electroencephalogram (EEG) was recorded by the usual routine settings for about one hour. EEG revealed bilateral spike and slow wave complexes during the recording on admission (Figure 1A). We treated the patient with $1000-1500 \mathrm{~mL} /$ day saline infusion and intravenous $5 \mathrm{mg}$ of diazepam, slowly injected without EEG recording; his consciousness improved temporarily, but became disturbed again. The next day his serum sodium improved to $133 \mathrm{mEq} / \mathrm{L}$. We confirmed irregular spike and slow wave complexes, and during drowsy and light sleep some focal spikes in the left anterior area (Figure 1B). On the third day, the spike and slow wave complexes disappeared. His conscious disturbance improved, as shown in EEG changes. After he recovered from disturbed consciousness, he had amnesia and could not remember anything from one to two days before his admission. Magnetic resonance imaging (MRI) showed small lacunars in the left basal ganglia and slight atrophy in the left temporal area. Single photon emission tomography (SPECT) during the interictal period showed an area of hypoperfusion in the left frontal and anterior-temporal area. Neuropsychological examination showed that Mini-Mental Status Examination and Raven Colored Progressive Matrices were 27/30 and 24/36, respectively, when the patient was alert. After admission we suspected drug-induced polydipsia, and stopped other medications apart from benzodiazepine, brotizolam $0.25 \mathrm{mg}$, and flunitrazepam $1 \mathrm{mg}$ for his insomnia. Thereafter, however, the patient often suffered from insomnia and had further difficulty controlling the polydipsia. Two weeks after admission, serum sodium, $\mathrm{ADH}$, and serum osmolarity were at normal levels. Therefore we diagnosed the patient as NCSE, as a result of polydipsiainduced hyponatremia. He was discharged in June X year.

The second episode occurred in August in X year. The patient vomited and had consciousness disturbance during the previous night. He came to our hospital by ambulance and was admitted in the early morning. On admission, serum

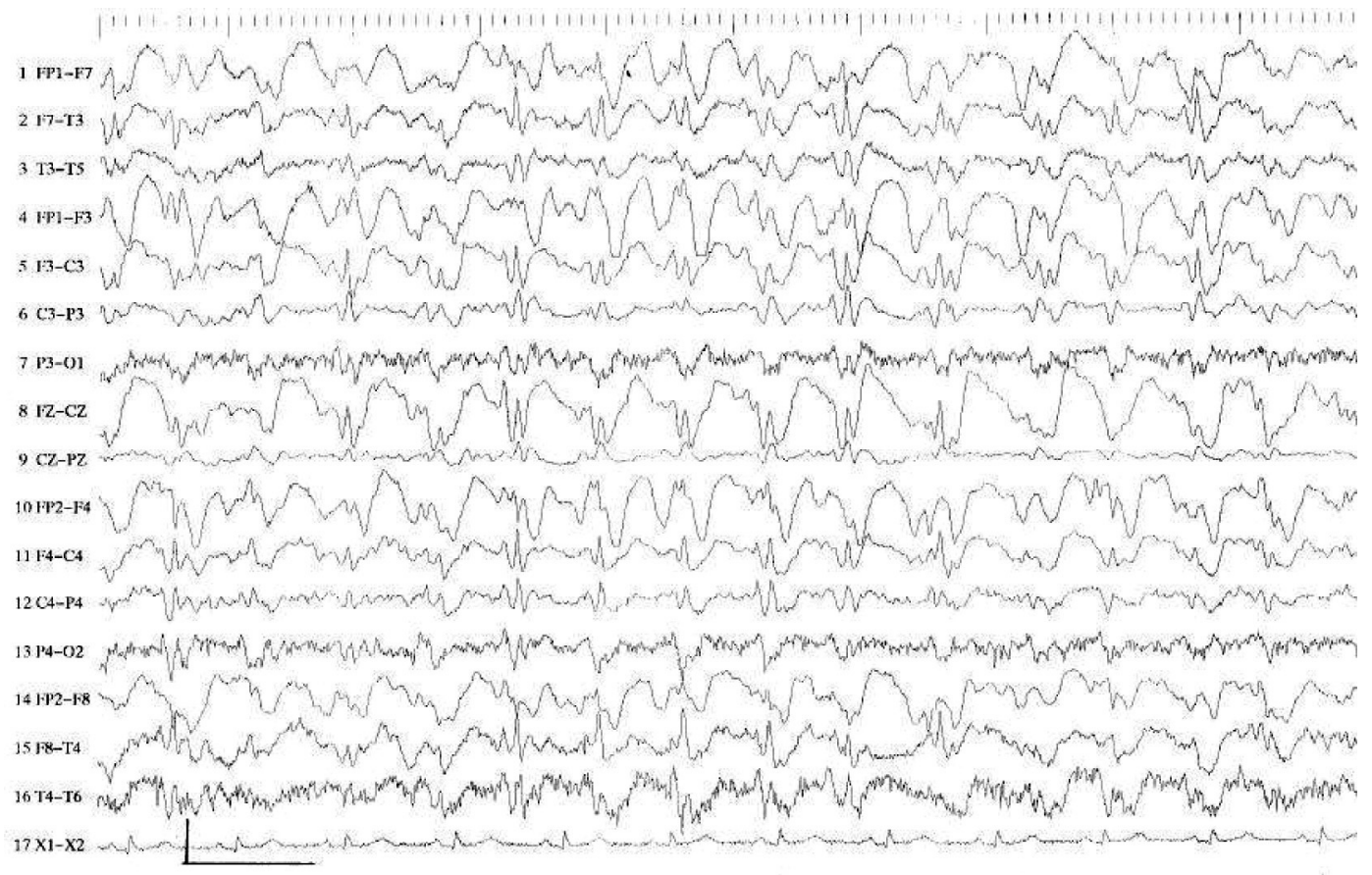

Figure IA On the first day of the first episode, continuously spike and slow wave complexes were seen in frontal area. The patient consciousness was disturbed. 
sodium was $126 \mathrm{mEq} / \mathrm{mL}$. We administered saline infusion $1500 \mathrm{~mL} /$ day. The next morning, the patient's serum sodium and consciousness had recovered. EEG revealed irregularly spike and slow wave complexes on the first day. But on the seventh day EEG revealed some spikes in the left anterior area. We did not use diazepam during this episode.

We considered that hyponatremia indirectly induced the onset of NCSE through the epiletpogenic zone in the left front-temporal area, and assumed that an anticonvulsant would be needed for NCSE. Thus we used phenytoin $200 \mathrm{mg}$ after the second episode, and the patient has had no recurrence of seizures and episodes of hyponatremia for five years.

\section{Discussion}

We diagnosed this case as NSCE, specifically, classical de novo absence status (Niedermeyer and Ribeiro 2000). Our patient had two absence status episodes and improved with the administration of sodium. EEG revealed continuously bilateral spike and slow wave complexes in the frontal area in both episodes, and showed some spikes in the left frontal area after recovery from absence status. Researchers have reported that benzodiazepines are effective for treating the clinical manifestations and EEG paroxysmal activity associated with absence status (Niedermeyer and Ribeiro 2000). We used benzodiazepine in the first episode, but we felt that correcting sodium levels was more clearly effective, because his consciousness disturbance was not clearly improved by diazepam in the first episode. Primavera and colleagues (1995) reported a case of de novo absence status with no focal signs in repeated episodes, which was treated effectively by correcting sodium levels.

Our case was de novo absence status showing continuously symmetrical frontal spike and slow wave complexes. After recovery from absence status, EEG showed some spikes in the left front-temporal area, MRI showed lacunar infarctions in the left basal ganglia, and SPECT showed hypoperfusion in the left anterior-frontal area. However, we clearly could not affirm the etiology of this region, for example, congenital or cryptogenic. We could not confirm that the onset of absence status started from this focal region, but suspected the left front-temporal region to be an epileptogenic zone. Kudo and colleagues (1995) reported that complex partial status mimicking absence status originates from the frontal area in unclassified NCSE. And Millan and colleagues (2001) reported cases of frontal localization of absence seizure. As a matter of course, absence seizure and absence status are distinct conditions that should not be considered together. However we considered that hyponatremia induced by polydipsia precipitated epileptogenicity in the left frontal area, and then focal activity secondarily generalized and resulted in absence status.

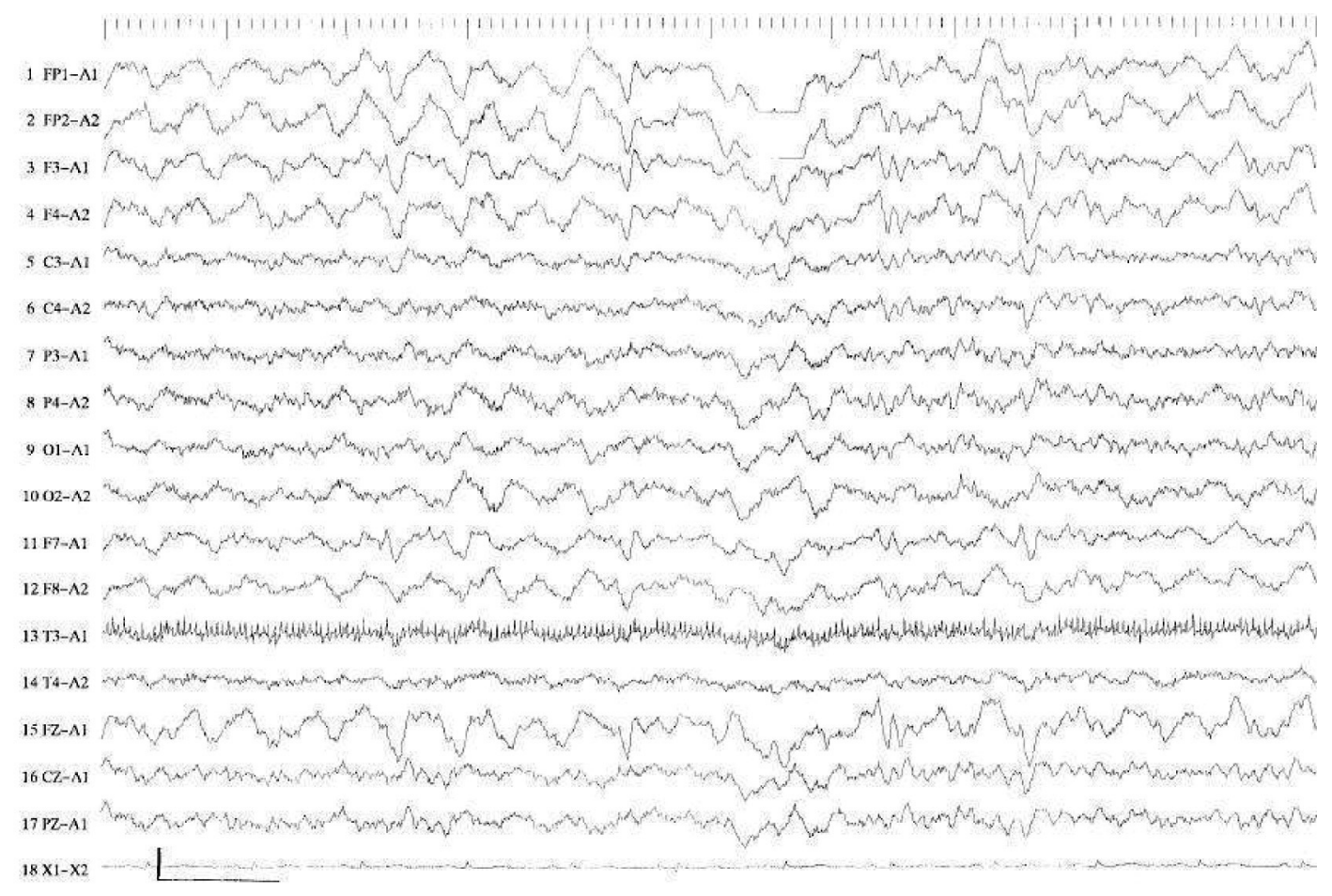

Figure IB On the second day of the first episode, some spikes were seen in left front-temporal area during drowsy state. The patient consciousness was almost normal. 
In conclusion, we reported a case of de novo absence status associated with two rare factors: a region of focal activity suspected of being an epileptogenic zone, and hyponatremia caused by polydipsia. In this case, the metabolic imbalance of hyponatremia, and the suspected left front-temporal epileptogenic zone, appeared to be involved in the onset of absence status. The answer to the controversy over the onset of NCSE awaits the elucidation of the origin of generalized spike and slow wave complexes.

\section{Disclosure}

We have no sources of support that require acknowledgment and we report no conflicts of interest.

\section{References}

Kudo T, Sato K, Yagi K, et al. 1995. Can absence status epilepticus be of frontal lobe origin? Acta Neurol Scand, 92:472-7.

Lee SI. 1985. Nonconvulsive status epilepticus. Ictal confusion in later life. Arch Neurol, 42:778-81.

Millan E, Abou-Khalil B, Delbeke D, et al. 2001. Frontal localization of absence seizures demonstrated by ictal positron emission tomography. Epilepsy Behav, 2:54-60.

Niedermeyer E, Ribeiro M. 2000. Considerations of nonconvulsive status epilepticus. Clin Electroencephalogr, 31:192-5.

Primavera A, Fonti A, Giberti L, et al. 1995. Recurrent absence status epilepticus and hyponatremia in a patient with polydipsia. Biol Psychiatry, 38:189-91.

Thomas P, Beaumanoir A, Genton P, et al. 1992. 'De novo' absence status of late onset: report of 11 cases. Neurology, 42:104-10.

Tomson T, Lindbom U, Nilsson BY. 1992. Nonconvulsive status epilepticus in adults: thirty-two consecutive patients from a general hospital population. Epilepsia, 33:829-35. 
